\title{
REVIEW
}

\section{New Drug Delivery Systems Concept in Anaesthesia and Intensive Care-Controlled Release of Active Compounds}

\author{
Anca Dinu ${ }^{1}$, Mihai Sandesc ${ }^{1}$, Sonia Elena Popovici², Razvan Gabriel Dragoi ${ }^{1}$, \\ Amaricai Elena1, Tiberiu Bratu ${ }^{1}$ \\ ${ }^{1}$ Faculty of Medicine, "Victor Babes" University of Medicine and Pharmacy, Timisoara 300041, Romania; \\ ${ }^{2}$ Clinic of Anaesthesia and Intensive Care, Emergency County Hospital "Pius Brinzeu", Timisoara \\ 300723, Romania;
}

\section{Correspondence to:}

Tiberiu Bratu, Pediatric Department, Faculty of Medicine, "Victor Babes" University of Medicine and Pharmacy, Timisoara,

Contact address: Romania, Eftimie Murgu 2, Timisoara, Romania

E-mail: tiberiu.bratu@umft.ro

\section{Conflicts of interests}

Nothing to declare

\section{Acknowledgment}

This article has been published with the help from the "Aurel Mogoseanu" Association for Anesthesia and Intensive Care, Timisoara, Romania. The authors wish to thank Clinical Research Network SRL Company for the support given in the completion of this paper.

Funding: This research did not receive any specific grant from funding agencies in the public, commercial or not-for profit sectors.

Keywords: drug release; liposomes; controlled release.

These authors take responsibility for all aspects of the reliability and freedom from bias of the data presented and their discussed interpretation.

Central Eur J Clin Res 2019;2(1):3-10

Received: 11.10.2018, Accepted: 5.01.2019, Published: 10.03.2019

Copyright $(2019$ Central European Journal of Clinical Research. This is an open-access article distributed under the Creative Commons Attribution License, which permits unrestricted use, distribution, and reproduction in any medium, provided the original work is properly cited.

\section{Abstract}

With time, medical and pharmaceutical research has advanced significantly. However, one of the major issues is how to administer the active substance. Among these, it counts overor under-dosage of the active substance, low response to treatment, or increased clinical risk of the patient. An innovative method able to avoid these obstacles is represented by controlled release systems for active substances. The interest for these systems came with allowing encapsulation in the antibiotic release matrices, local anesthetics, protein or other substances. Moreover, a number of such vehicles are now available to release controlled substances used predominantly in the anesthesia and intensive care unit.

\section{Introduction}

A lot of biologically active compounds have been discovered with the developments 
in science and research, compounds that can be ideal candidates for the treatment of diverse pathologies. The biological activity depends on the way of administration and on the type of metabolism a certain substance has inside the human body. Active substances administered orally have a pretty low bioavailability, and therefore supplementary administration of the drug is needed at set time intervals, resulting in a lower efficiency, in the risk of underdosage or overdosage, and into a higher discomfort for the patient [1]. Recently new materials have come to light, that are capable of encapsulating the active biological compound in a polymer network and of delivering it into a system (biological or physic-chemical), in a constant manner, and over long periods of time. Much has been taken of the functionalized materials-capable of reaching a target place in the body and to precisely control further aspects: the volume of the entrapped molecule and the concentration of the biologically active compound, resulting in a new field of research: Controlled Drug Release-controlled drug delivery. The chemical materials research field needed for manufacturing the drugs with controlled release is continuously developing because of very strict parameters that need to be met-bioavailability, stimulus response, biodegradability, etc. The release speed of active biological compounds from the systems obtained through various methods can be adapted based on the clinical needs for the treatment by changing certain chemical compounds in the manufacturing process, and therefore reaching the expected therapeutical requirements $[1,2]$. Controlled release systems from biologically active compounds are devices that allow the administration inside of the body of an active substance, by incorporating it inside them and improving their therapeutic effect by controlling the speed, target place and time of release [3]. Compared to conventional systems, the ones with controlled release allow maintaining an optimal concentration of the active compound that stays between therapeutic limits, without reaching toxic levels and without it being under dosed. The results of experimental studies stand proof for the importance of introducing controlled release systems in the current medical practice [4]. The diversity of controlled release systems can be increased through the biofunctionalizing of particles that are part of the material used when manufacturing the system. In this manner the following can be grafted on the structures of controlled release systems: proteins, lipids, nucleic acids, active substances, and biomacromolecules [5]. The aim of this paper is that of creating a link between poly- meric biocompatible and bioavailable matrices used for the release of certain biologically active compounds and treatments of interest in the anesthesia and intensive care units.

\section{Types of Materials Used in Manufac- turing-Controlled Release Systems}

Because of the high diversity regarding the clinical needs, numerous studies have been carried out in the field of biocompatible materials that could attain the selectivity, metabolism, bioavailability, and biodegradability requirements. Therefore, numerous polymer networks have been developed, that can be successful candidates for carrying biologically active compounds that are of clinical interest. In the following pages we will present some of the materials used for manufacturing-controlled release systems.

\section{Chitosan-Based Hydrogels}

Hydrogels are part of the "intelligent" controlled release systems because of their properties: biodegradability, functionality, biorecognition, and permeability [4]. Hydrogels are reticular networks with a high number of hydrophilic groups, with high affinity for water, allowing it to get inside the polymer network. The best results were obtained with hydrogels manufactured out of chitosan because of the body's ability to degrade these structures through enzymatic metabolism [5]. Chitosan is a linear polysaccharide made out of $\beta$ units $-\beta$ (1-4)-D-glucosamine and $\mathrm{N}$-acetic-D-glucosamine, is the ideal candidate for controlled release systems and it is also ranked first in other studies in the field of non-viral vectors such as for DNA genes [5,6]. Because it is non-toxic, stable, biodegradable, and can be sterilized, it can be used for synthetizing different types of hydrogels: liquid gels, powder, films, tablets, microspheres, nanofibers etc. For manufacturing hydrogels one can also use synthetic hydrophilic polymers (poly- $\mathrm{N}$-isopropylacrylamide, polyvinyl alcohol), but not being fully biodegradable they can lead to local inflammation. Polymers from hydrogels can absorb different quantities of water that can vary from o fraction to thousand times their own mass, depending on the hydrophilic groups present in the network. This physical phenomenon leads to the gel's expansion, which will therefore have common properties with the surrounding live tissues-low superficial pressure, soft consistency. Polymer networks are formed in the presence of the biologically active compound and lead to its encapsulation. The biofunctionalization of the 
chitosan-based hydrogels increases their bioavailability though the formation of bionanomaterials with new functions. In 2014, Roberio et al. [7] have synthetized a series of bio hybrids, through the functionalization of crab shell extracted chitosan (CHT) with pectin (PCT) extracted from fruit juice and double layer hydroxides $(\mathrm{LDH},[\mathrm{Mg0}, 67 \mathrm{Al0}, 33(\mathrm{OH}) 2] \mathrm{Cl0}, 33 \times \mathrm{n}$ $\mathrm{H} 2 \mathrm{O}$-obtained after the Constantino and Pinnava method from 1998) [7]. Hence, they have obtained a bio-nanomaterial, CHT/LDS-5ASA [7], with new functions that can serve as vehicle for controlled release system used in clinical practice. The release of the active compound is influenced by a series of physical and chemical processes: diffusion, expansion or enzymatic degradation [8,9]. Expansion leads to pores "opening" and to the release of the active substance, while enzymatic degradation destroys the networks and, therefore, depending on the mechanism, leads to the release of the active compound in the system. For example, the release of the biologically active compound based on the biochemical degradation mechanisms of the matrix has the advantage of bioselectivity $[7,9]$.

Choosing the correct material type for the manufacturing of the hydrogel, choosing the type of network and the type of release mechanisms assures a series of pharmacological qualities of the active substance during therapy.

\section{Sol-Gel Siloxane Matrices}

The sol-gel process is based on the ability of some hydrolabile precursors to form metal or semi-metal solid oxides through aqueous processing [10]. The pore-like gel is obtained through the condensation and polycondensation of hydroxylated units obtained through the hydrolysis of a precursor in an acidic or basic environment $[10,11]$. The silanol $(\mathrm{Si}-\mathrm{OH})$ groups will be formed, that through condensation will lead to the formation of siloxanes (-Si-O-Si-), and through maturation and drying lead to the formation of the siloxane matrix. The matrix that will results is a rigid network with pores of under one micrometer and polymer chains with a medium length of over a micrometer [11]. The direct immobilization of the active substance (enzymes, antibodies, proteins, DNA, and ARN) will lead to the physical entrapment that can take place due to the specific properties of siloxane matrices: adjustable porosity, chemical persistency, mechanical and optical stability, normal expandability, thermic stability [12]. Encapsulating the biomolecules without altering their initial conformation represents the main advantage of the controlled release systems; this property exists due to the recesses that ensure a rigid environment for the encapsulated molecule [13,14]. The size and density of the pores are controlled rigorously during the synthesis in order to eliminate the eventual blockage of molecules inside the matrix, fact that could lead to the alteration of the therapeutic effect [15]. Depending on the precursors, one can obtain different types of matrices (inorganic or hybrid) with different properties: functionality, superficial pressure, controllable porosity, and increased mechanical thermic stability [15-17]. Preda et al. in a study on the entrapment of alkalasys in a sol-gel matrix [16], have analyzed the siloxane matrix with the entrapped enzyme thermogravimetrically, in different thermic phases. They have shown that after $500{ }^{\circ} \mathrm{C}$ the loss of mass is justified by the total dehydroxilation and by total destruction of the organic compounds, including the enzyme, with a total mass loss of $23 \%$ - confirmed by the results from IR analysis. This study has proven the high stability of siloxane matrices capable of entrapping the active substance, and capable of functioning as vehicles for controlled release for the active principle in a system [16].

\section{Amphiphilic Dendrimers}

The monomers that are organized under a tree-like shape, around a central nucleus, lead to the appearance of macromolecules called dendrimers $[18,19]$. Jansen et al. have proven that in the case of dendrimers the immobilization/release of the active compound in the matrix takes place because its specific steric properties [18,20,21]. Dendrimer synthesis is based on two methods: divergent and convergent [21]. Through the divergent method the monomers are assembled from the nucleus towards the periphery, while through the convergent method they are synthetized from the periphery towards the nucleus through some fragments called dendrons. Depending on the number and diameter of the monomers there are different generations of dendrimers (e.g., generation 0-G0, $1.4 \mathrm{~nm}$; generation 4-G4, $4.4 \mathrm{~nm}$ ). Because of they are multifunctional and due to their particular characteristics, dendrimers can transport biomolecules (chromosomal elements, cytostatic drugs) towards target areas in the body $[18,22]$. Dendrimers can be functionalized through metal grafts ( $\mathrm{Au}$, $\mathrm{Ag}, \mathrm{Ru}, \mathrm{Rh}$ ) on the surface of the structure or through grafts of different other molecules (polyethylene glycol, t-butoxycarbonyl) [23]. 


\section{Carbon Nanotubes}

Carbon nanotubes are used especially in the oncology field, in cytostatic therapies. Carbon nanotubes are carbon allotropes with a cylindrical structure [23-26]. Their special properties are given by their biofunctionalization with biomolecules, transforming them in ideal systems for controlled release of active biological compounds $[24,26,27]$. The development of their application in the field of nano-medicine has been motivated through the fact that bionanostructures are capable of penetrating the cell and of delivering to target the active principle, especially for substances with a low molecular mass $[26,28,29]$.

\section{Cyclodextrines}

Cyclodextrines are cyclic oligosaccharides that form macromolecules with tridimensional structures incorporating a high number of exterior hydroxyl groups $[1,30]$. This structure gives them a hydro soluble exterior structure and a hydrophobic interior, used for the entrapment of the active principle [30,31]. Depending on the composition and on the proportion of saccharide structures there are three different types of cyclodextrines: $\alpha-, \beta-, \gamma-[32,33]$.

\section{Controlled Release Mechanisms}

The controlled release mechanisms that are characteristic for the systems used in clinical treatments are divided in two categories: physical mechanisms and chemical mechanisms [32]. In the case of physical mechanisms, the release kinetics is only controlled by the structure of the polymer network. In this case the activity of the release system can be increased by adjusting some simple parameters during the manufacturing process, or by choosing a certain type of material. Chemical mechanisms are based on the chemical bonds that grow between the polymer network and the active biological compound, but present the disadvantage that the structure of the active compound is modified, resulting chemical entities that can have unwanted effects [33,34]. For a higher efficacy of the active substance a certain, optimal concentration in the blood (therapeutic index) should be maintained. The therapeutic index $(\mathrm{TI})$ represents the ratio between the maximum admitted concentration (Cmax) and the minimal concentration required for action of the drug (Cmin), and therefore $\mathrm{TI}=\mathrm{Cmax} /$ Cmin. Each active substance has a specific TI, for example TItrifenilalanina $=20,000$, iar Tldig- itoxin $=2$, case in which the value of the therapeutic index become highly valuable $[30,33,35]$. Among the most widely used physical mechanisms for controlled release is the degrading/ dissolving and ion exchange. Degrading or dissolving refers to the destruction of the polymer structure that encapsulates the biologically active compound. The release speed of the biomacromolecules, and implicitly of the polymer structure depends on the water solubility of the polymer [36]. Therefore, the polyglycolic acid and polycaprolactone form networks that, because of low water solubility, are degraded in weeks, or even months-therefore is a rapid release is wanted; one should use materials with high water solubility. The ion exchange I used especially for active substances that have a permanent ion character, that are caught in the polymer network through electrostatic interactions. The biomacromolecule's ion is replaced with another ion, with the same electrical charge in the polymer network, and its delivery to the tissue is hence possible $[25,37,38]$.

\section{The Applicability of Controlled Release Systems to Anesthesia and Intensive Care Units}

\section{Transdermal Fentanyl Absorption}

Transdermal absorption of active substances offers a series of advantages over the classical administration routes because they are absorbed slowly, in a constant concentration, and for longer periods of time [39]. A series of substances are still administered transdermal, among which we find the following: nitroglycerine, scopolamine, clonidine, lidocaine, estradiol, or fentanyl [40]. The active compound is stored inside the therapeutic transdermal system (TTS), and through the application of the patch the delivery of molecules will begin due to the difference in concentration gradients. Administered through TTS, Fentanyl acts in a minimally invasive manner, after a well-known kinetics, leading to increased patient satisfaction. The molecular weight of the active substance (337 Da), that does not overcome the maximal molecular weight adequate for skin permeability (1000 Da), as well as the fact that fentan$\mathrm{yl}$ is soluble in the lipid compartments of the skin (600 times more soluble than morphine), determines a continuous diffusion of the active compound in a safe, non-invasive manner that offers the patient increased comfort during the therapy [41,42]. There are different systems designed for the controlled release of fentanyl: TTS fentanyl-25 that releases $25 \mu \mathrm{g} / \mathrm{h}$, TTS 
fentanyl-100 that releases $100 \mu \mathrm{g} / \mathrm{h}$, and other doses. The delivery system comes as an adhesive patch (reservoir/matrix in which fentanyl is dissolved in ethanol) that will be applied on the skin and will be capable of delivering an optimal opioid concentration for as long as $72 \mathrm{~h}$ $[39,43,44]$. Fentanyl becomes detectable in serum after approximately $60-120$ min since the application of the patch and reaches its maximal concentration after $15 \mathrm{~h}$. Depending on the system type, the fentanyl serum concentration can reach $2.6 \mathrm{ng} / \mathrm{mL}$ for TTS fentanyl-100 [45-48]. Kress et al. have studied the plasma concentration of fentanyl after applying different types of transdermal release patches $(100 \mu \mathrm{g} / \mathrm{h})$, and they observed that the concentration values for the active substance are equivalent with one another [49]. The clinical effects of fentanyl depend on the dose and on the patient's tolerance to opioids. Transdermal opioid release has proven very efficient in the long term treatment of patients suffering from neoplastic pain, but is also used in the treatment of acute postoperative pain, as well as in other forms of chronic pain $[44,45]$.

\section{Local Anesthetics with Controlled Release}

Controlled release systems for local anesthetics have been developed in order to avoid various accidents, in order to assure a longer time of action for local anesthetics (days or weeks), and for avoiding certain methods used nowadays (e.g., epidural catheters that can lead to serious infections) $[44,48]$. Liposomes, dextrines, cyclodextrines, and biopolymers have all been tested for use in the formulation of such systems capable of releasing local anesthetics for longer periods of time.

\section{Liposomes}

Liposomes are made out of lipid or phospholipid molecules with a hydrophilic (head) and hydrophobic (tail) part. They are of great importance because the liposomal mycelia can be encapsulated in concentrated solutions of local anesthetics $[45,46,49]$. They are biocompatible, biodegradable, and non-immunologic, allowing gradual release of the anesthetic during the slow degradation of the liposomal mycelia. Using these new compounds reduces the systemic toxicity, as well as other adverse effects $[48,50]$. The increased bioavailability of these liposomal compounds was achieved through polyethilenglycol grafts. The liposomes functionalized with this technique $[25,48]$ have significant properties for the interaction with proteins and human cells, and have an important impact on the capacity of controlled release of local anesthetic $[48,50,51]$. Ilfeld et al., have carried out a femoral block in 14 healthy volunteers using liposomal bupivacaine in different doses (EXPAREL, Pacira Pharmaceuticals, Inc., San Diego, CA, USA) obtaining a variable sensitive blockade and a motor blockade that lasted for over $24 \mathrm{~h}$, for the maximal used dose [52]. Viscusi et al. have studied the pharmacokinetics, sensitive and motor effects for the epidural administration of liposomal bupivacaine (89 mg, $155 \mathrm{mg}, 266 \mathrm{mg}$ ) versus $\mathrm{HCl}$ bupivacaine $(50 \mathrm{mg})$ [53]. The study has shown that though the administration of liposomal bupivacaine $266 \mathrm{mg}$, the sensitive blockade lasted longer, in comparison to the motor blockade that lasted longer when $\mathrm{HCl}$ bupivacaine $50 \mathrm{mg}$ was administered. Slow release bupivacaine was tolerated well when administered epidural, with local pain at injection as side effect that disappeared after 30 days for the majority of the subjects in the study group [53].

\section{Cyclodextrines}

Another type of chemical compounds that can be used for the controlled release local anesthetics are cyclodextrines. $\beta$-cyclodextrine has a positive impact on increasing bioavailability and decreasing toxicity for local anesthetics. Combining the anesthetics agents (especially bupivacaine and ropivacaine) with cyclodextrines leads to the formation of an extremely advantageous system, offering new properties to the local anesthetic-increased solubility in an aqueous environment, increased chemical stability, and new pharmacodynamic properties. The levobupivacaine-maltozyl- $\beta$-cyclodextrine has decreased neurotoxicity of anesthetic agents after spinal anesthesia. There are numerous studies on the combination of anesthetic agents as basic substances or as salts in concentrations of $0.02 \%$ with a-ciclodextrine, $\beta$-ciclodextrine sau $y$-ciclodextrine $[33,50,51]$. Therefore, numerous systems have been obtained, capable of controlled and gradual release of the local anesthetic-gels, oils, suppositories, or patches. Local anesthetic agents can be associated with other active substances-antihistamines, adrenocorticosteroids etc. $[33,50,54]$.

\section{Biopolymers}

A biopolymer with high bioavailability in the human body is the biopolymer formed from sebacic acid and ricinoleic acid in a 2:8 molar 
ratio $[55,56]$. Shikanow et al. have carried out numerous in vitro and in vivo studies on the encapsulation of bupivacaine in a biopolymer, following its release kinetics. The obtained results confirm that controlled release bupivacaine systems can have a longer duration of action, up to 14 days [55]. In parallel they have carried out in vivo studies, sciatic nerve block in lab rats, using the bupivacaine-polymer complex. The study animals were injected with $0.1 \mathrm{~mL}$ of the bupivacaine-polymer complex $10 \% \mathrm{w} / \mathrm{w}$, while for the control they administered the polymer without bupivacaine on the opposite side. In this manner they studied the efficacy, metabolism and elimination of bupivacaine. The controlled release system brought impressive results, leading to an $18 \mathrm{~h}$ long motor block $[45,55,56]$. There are numerous natural or synthetic polymer mixtures, bioavailable and biocompatible, that can form controlled release systems for local anesthetics-polycaprolactone, polyactide, polyesters, hydroxipropyl cellulose, N-isopropylacrilamide, polyvinyl alcohol etc.) $[3,55,56]$. These polymer systems (micro- and nanoparticles) can exist under different forms-spheres, capsules [56]. The nanoparticles and microparticles in which the active substances are encapsulated considerably reduce the systemic toxicity of local anaesthetics.

\section{Conclusions}

Controlled release systems have drawn attention because of the advantages they can bring to clinical practice. In the field of anesthesia these new discoveries have resulted in the encapsulation of local anesthetic agents and opioids in controlled release matrices, that can significantly reduce the adverse effects associated with the classical administration routes, contributing to drug delivery safety and patient satisfaction.

\section{References}

1. Zhang $\mathrm{Y}$, Chan HF, Leong KW. Advanced materials and processing for drug delivery: the past and the future. Adv Drug Deliv Rev 2013; 65:104-120

2. Mrsny RJ. Oral drug delivery research in Europe. J Control Release 2012; 161:247253

3. Webster DM, Sundaram P, Byrne ME. Injectable nanomaterials for drug delivery: carriers, targeting moieties, and therapeutics. Eur J Pharm Biopharm 2013; $84: 1-20$

4. Ukmar T, Maver U, Planinšek O, Kaučič
V, Gaberšček M, Godec A. Understanding controlled drug release from mesoporous silicates: Theory and experiment. Journal of Controlled Release 2011; 155:409-417

5. Rana S, Bajaj A, Mout R, Rotello VM. Monolayer coated gold nanoparticles for delivery applications. Adv Drug Deliv Rev 2012; 64:200-216

6. Castro E, Mosquera V, Katime I. Dual Drug Release of Triamterene and Aminophylline from Poly(N-isopropylacrylamide) Hydrogels. Nanomater Nanotechnol 2012; 2:1-9

7. Robeiro LN, Alcântara AC, Darder M, Aranda P, Araújo-Moreira FM, RuizHitzky E. Pectin-coated chitosan-LDH bionanocomposite beads as potential systems for colon-targeted drug delivery. Int J Pharm 2014; 463:1-9

8. Wang C, Ye W, Zheng Y, Liu X, Tong Z. Fabrication of drug-loaded biodegradable microcapsules for controlled release by combination of solvent evaporation and layer-by-layer self-assembly. Int J Pharm 2007; 338:165-173

9. Ma J, Zhang M, Lu L, Yin X, Chen J, Jiang Z. Intensifying esterification reaction between lactic acid and ethanol by pervaporation dehydration using chitosan-TEOS hybrid membranes. Chem Engin J 2009; 155:800809

10. Morpurgo M, Teoli D, Pignatto M, Attrezzi M, Spadaro F, Realdon N. The effect of $\mathrm{Na} 2 \mathrm{CO} 3, \mathrm{NaF}$ and $\mathrm{NH} 4 \mathrm{OH}$ on the stability and release behavior of sol-gel derived silica xerogels embedded with bioactive compunds. Acta Biomater 2010; 6:22462253

11. Estella J, Echeverría JC, Laguna M, Garrido JJ. Effects of aging and drying conditions on the structural and textural properties of silica gels. Micropor Mesopor Mater 2007; 102:274-282

12. Tsai $\mathrm{CH}$, Lin HJ, Tsai HM, Hwang JT, Chang SM, Chen-Yang YW. Characterization and PEMFC application of a mesoporous sulfonated silica prepared from two precursors, tetraethoxysilane and phenyltriethoxysilane. Int $\mathrm{J}$ Hydrogen Energy 2011; 36:9831-9841

13. Estella J, Echeverría JC, Laguna M, Garrido JJ. Silica xerogels of tailored porosity as support matrix for optical chemical sensors. Simultaneous effect of $\mathrm{pH}$, ethanol:TEOS and water:TEOS molar ratios, and synthesis temperature on gelation time, and textural and structural properties. J NonCryst Solids 2007; 353: 286-294 
14. Timin AS, Rumyantsev EV. Sol-gel synthesis of mesoporous silicas containing albumin and guanidine polymers and its application to the bilirubin adsorption. J Sol-Gel Sci Technol 2013; 67:297-303

15. Radin S, Chen T, Ducheyne P. The controlled release of drugs from emulsified, solgel processed silica microspheres. Biomaterials 2009; 30: 850-858

16. Dudás Z, Chiriac A, Preda G. Simple entrapment of alcalase in different silica xerogels using the two steps sol-gel method. Annals of West University of Timişoara - Series Chemistry 2011; 20:97104

17. Tan S, Wu Q, Wang J, et al. Dynamic selfassembly synthesis and controlled release as drug vehicles of porous hollow silica nanoparticles. Micropor Mesopor Mater 2011; 142:601-608

18. Wang Y, Grayson SM. Approaches for the preparation of non-linear amphiphilic polymers and their applications to drug delivery. Adv Drug Deliv Rev 2012; 64:852865

19. Prow TW, Grice JE, Lin LL, et al. Nanoparticles and microparticles for skin drug delivery. Adv Drug Deliv Rev 2011; 63: 470-491

20. Jansen JFGA, Meijer EW, de Brabandervan den Berg EMM. The dendritic box: shape-selective liberation of encapsulated guests. J Am Chem Soc 1995; 117:44174418

21. Venkataraman S, Hedrick JL, Ong ZY, et al. The effects of polymeric nanostructure shape on drug delivery. Adv Drug Deliv Rev 2011; 63:1228-1246

22. Mudshinge SR, Deore AB, Patil S, Bhalgat CM. Nanoparticles: Emerging carriers for drug delivery. Saudi Pharm J 2011; 19:129141

23. Prakash S, Malhotra M, Shao W, TomaroDuchesneau C, Abbasi S. Polymeric nanohybrids and functionalized carbon nanotubes as drug delivery carriers for cancer therapy. Adv Drug Deliv Rev 2011; 63:1340-1351

24. Han G, Ghosh P, De M, Rotello VM. Drug and gene delivery using gold nanoparticles. Nanobiotechnol 2007; 3:40-45

25. Janib SM, Moses AS, MacKay JA. Imaging and drug delivery using theranostic nanoparticles. Adv Drug Deliv Rev 2010; 62:1052-1063

26. Lee KY, Yuk SH. Polymeric protein delivery systems. Prog Polym Sci 2007; 32:669-697

27. Suh WH, Suslick KS, Stucky GD, Suh
YH. Nanotechnology, nanotoxicology, and neuroscience. Prog Neurobiol 2009; 87:133-170

28. Portney NG, Ozkan M. Nano-oncology: drug delivery, imaging, and sensing. Anal Bioanal Chem 2006; 384:620-630

29. Zalfen AM, Nizet $D$, Jérôme $C$, et al. Controlled release of drugs from multicomponent biomaterials. Acta Biomater 2008; 4:1788-1796

30. Cheng S, Song Q, Wei D, Gao B. Highlevel production penicillin $G$ acylase from Alcaligenes faecalis in recombinant Escherichia coli with optimization of carbon sources. Enzyme Microb Technol 2007; 41:326-330

31. Wang F, Saidel GM, Gao J. A mechanistic model of controlled drug release from polymer millirods: effects of excipients and complex binding. Control Release 2007; 119:111-120

32. Machín R, Isasi JR, Vélaz I. Hydrogel matrices containing single and mixed natural cyclodextrins. Mechanisms of drug release. Eur Polym J 2013; 49: 3912-3920

33. Kona S, Dong JF, Liu Y, Tan J, Nguyen KT. Biodegradable nanoparticles mimicking platelet binding as a targeted and controlled drug delivery system. Int J Pharm 2012; 423:516-524

34. Krishnamachari Y, Geary SM, Lemke CD, Salem AK. Nanoparticle delivery systems in cancer vaccines. Pharm Res 2011; 28:215236

35. Xia W, Chang J, Lin J, Zhu J. The pHcontrolled dual-drug release from mesoporous bioactive glass/polypeptide graft copolymer nanomicelle composites. Eur J Pharm Biopharm 2008; 69:546-552

36. Kreye F, Siepmann F, Siepmann J. Drug release mechanisms of compressed lipid implants. Int J Pharm 2011; 404:27-35

37. Tran PHL, Choe JS, Tran TTD, Park YM, Lee BJ. Design and mechanism of onoff pulsed drug release using nonenteric polymeric systems via $\mathrm{pH}$ modulation. AAPS PharmSciTech 2011; 12:46-55

38. Hayashi $T$, Kanbe $H$, Okada $M$, et al. Formulation study and drug release mechanism of a new theophylline sustained-release preparation. Int J Pharm 2005; 304: 91-101

39. Yoshida T, Tasaky H, Maeda A, Katsuma M, Sako K, Uchida T. Mechanism of controlled drug release from a salting-out tastemasking system. J Control Release 2008; 131:47-53

40. Fredenberg S, Wahlgren M, Reslow M, 
Axelsson A. The mechanisms of drug release in poly(lactic-co-glycolic acid)based drug delivery systems - A review. Int J Pharm 2011; 415:34-52

41. Ferrero C, Massuelle D, Doelker E. Towards elucidation of the drug release mechanism from compressed hydrophilic matrices made of cellulose ethers. II. Evaluation of a possible swelling-controlled drug release mechanism using dimensionless analysis. J Control Release 2010; 141: 223-233

42. Gustavsson A, Bjorkman J, Ljungcrantz C, et al. Pharmaceutical treatment patterns for patients with a diagnosis related to chronic pain initiating slow-release strong opioid treatment in Sweden. Pain 2012; 153:23252331

43. Hoya Y, Okamoto T, Yanaga K. Evaluation of analgesic effect and safety of fentanyl transdermal patch for cancer pain as the first line. Support Care Cancer 2010; 18:761-764

44. Kress HG, Von der Laage D, Hoerauf $\mathrm{KH}$, et al. A randomized, open, parallel group, multicenter trial to investigate analgesic efficacy and safety of a new transdermal fentanyl patch compared to standard opioid treatment in cancer pain. J Pain Symptom Manage 2008; 36: 268-279

45. Lane ME. The Transdermal delivery of fentanyl. Eur J Pharm Biopharm 2013; 84:449-455

46. Minkowitz HS. Fentanyl iontophoretic transdermal system: a review. Tech Reg Anesth Pain Managt 2007; 11: 3-8

47. Freynhagen $\mathrm{R}$, von Giesen $\mathrm{HJ}$, Busche P, Sabatowski R, Konrad C, Grond S. Switching from reservoir to matrix systems for the transdermal delivery of fentanyl: a prospective, multicenter pilot study in outpatients with chronic pain. J Pain Symptom Manage 2005; 30:289-297

48. Margetts L, Sawyer R. Transdermal drug delivery: principles and opioid therapy.
Contin Educ Anaesth Crit Care Pain 2007; 7:171-176

49. Kress HG, Boss $H$, Delvin $T$, et al. Transdermal fentanyl matrix patches Matrifen and Durogesic DTrans are bioequivalent. Eur J Pharm Biopharm 2010; 75:225-231

50. Bajaj S, Whiteman A, Brandner B. Transdermal drug delivery in pain management. Contin Educ Anaesth Crit Care Pain 2011; 11:39-43

51. Coulthard $P$, Oliver $R$, Khan Afridi KA, Jackson-Leech D, Adamson L, Worthington $H$. The efficacy of local anaesthetic for pain after iliac bone harvesting: a randomised controlled trial. Int J Surg 2008; 6:57-63

52. Ilfeld BM, Malhotra N, Furnish TJ, Donohue MC, Madison SJ. Liposomal bupivacaine as a single-injection peripheral nerve block: a dose-response study. Anesth Analg 2013; 117:1248-1256

53. Viscusi ER, Candiotti KA, Onel E, Morren M, Ludbrook GL. The pharmacokinetics and pharmacodynamics of liposome bupivacaine administered via a single epidural injection to healthy volunteers. Reg Anesth Pain Med 2012; 37:616-622

54. Tsuchiya H, Ueno T, Mizogami M, Takakura K. Local anesthetics structure-dependently interact with anionic phospholipid membranes to modify the fluidity. Chem Biol Interact 2010; 183:19-24

55. Shikanov A, Domb AJ, Weiniger CF. Long acting local anesthetic-polymer formulation to prolong the effect of analgesia. J Control Release 2007; 117: 97-103

56. Rodriguez-Navarro AJ, Lagos M, Figueroa $C$, et al. Potentiation of local anesthetic activity of neosaxitoxin with bupivacaine or epinephrine: development of a long-acting pain blocker. Neurotox Res 2009; 16:408415 